\title{
Modified Da Chengqi granules improvement in immune function in early severe acute pancreatitis patients
}

\author{
D.-L. Jiang ${ }^{1}$, J. Yang ${ }^{2}$, S.-Y. Jiang'1, F.-L. Yuan', Y.-L. Gu ${ }^{2}$, J.-P. Li ${ }^{2}$ and \\ Z.-J. Pei ${ }^{3}$
}

${ }^{1}$ Wuxi Institute of Integrated Traditional Chinese and Western Medicine, The Third Affiliated Hospital to Nantong University, Wuxi, Jiangsu Province, China

${ }^{2}$ Departament of Digestion,

Wuxi Hospital of Integrated Traditional Chinese and Western Medicine,

The Third Affiliated Hospital to Nantong University, Wuxi, Jiangsu Province, China

${ }^{3}$ Department of Pharmacy, Wuxi No. 2 People's Hospital, Wuxi, Jiangsu Province, China

Corresponding author: Z.-J. Pei

E-mail: jdlstar@126.com

Genet. Mol. Res. 15 (2): gmr.15028787

Received May 16, 2016

Accepted June 3, 2016

Published June 24, 2016

DOI http://dx.doi.org/10.4238/gmr.15028787

\begin{abstract}
We investigated the role of modified Da Chengqi granules in improving immune function in early severe acute pancreatitis patients. Early severe acute pancreatitis patients who agreed to receive combined treatment of traditional Chinese and Western medicine were randomly assigned to the experimental or control group. All subjects received conventional therapy to support organ function. The experimental group also received modified Da Chengqi granules. Cytokine (interleukin-6, interleukin-10, and tumor necrosis factor- $\alpha$ ) levels, immunological markers (HLA-DR, Treg, and Th1/Th2), urinary lactulose/mannitol ratio, and endotoxin levels were measured at 1,3,7, and 14 days after hospital
\end{abstract}


admission. The total mortality rate was $11.69 \%(9 / 77)$, which was significantly lower in the experimental group $[4.88 \%(2 / 41)]$ than in the control group $\left[19.44 \%(7 / 36) ; \chi^{2}=3.940, \mathrm{P}<0.05\right]$. Serum interleukin-6, interleukin-10, tumor necrosis factor- $\alpha$ and endotoxin levels and the lactulose/mannitol ratio were significantly lower on day 7 and day 14 than on day 1 in experimental and control groups $(\mathrm{P}<0.01)$. Immunological indices were significantly lower in the experimental group than in the control group on day 14 (all $\mathrm{P}<0.01$ or 0.05 ). HLA-DR-positive cell ratio gradually increased over 14 days in experimental and control groups $(\mathrm{P}<0.01$ vs day 1$)$, but was higher in the experimental group than in the control group by day $14(\mathrm{P}<0.05)$. Notably, Treg cell prevalence and Th1/Th2 cell ratio deteriorated within 7 days in both groups $(\mathrm{P}<$ $0.01 v s$ day 1$)$, but then returned to day 1 levels $(\mathrm{P}<0.01$ or $0.05 v s$ day 1). Significant differences in Treg levels and Th1/Th2 cell ratio between experimental and control groups were observed on day $14(\mathrm{P}<0.01)$. These results show that modified Da Chengqi granules can improve immune function in early severe acute pancreatitis patients.

Key words: modified Da Chengqi granules; severe acute pancreatitis; immune function; Chinese medicine

\section{INTRODUCTION}

Severe acute pancreatitis is a common abdominal condition characterized by acute onset, rapid progression, and complicated pathological changes. In the early phase, patients often suffer from systemic inflammatory response syndrome (SIRS) and multiple organ dysfunction syndrome (MODS) (Barie et al., 2009; Vlada et al., 2013). The mortality rate of severe acute pancreatitis is high (Barie et al., 2009; Vlada et al., 2013), especially in patients experiencing immunological imbalance. This imbalance includes changes in the activating factors of the immune system, changes in immunologically competent cells, and inhibition of intestinal immunologic function (Kylänpää et al., 2010; Dambrauskas et al., 2010; Shen et al., 2011). A key to the prevention and treatment of SIRS and MODS is to restore the immunological balance between the immunoregulatory cells, cytokines, and immune mediators. This also reflects the theory of global balance in Chinese medicine. In Chinese medicine, treatment is based on syndrome differentiation and aims to recover the global balance and treat the primary and secondary aspects of the disease at the same time (Wu, 2004; Fang et al., 2007). In this prospective study, we observed improvement in immune function in early severe acute pancreatitis patients receiving conventional therapy in combination with modified Da Chengqi granules.

\section{MATERIAL AND METHODS}

\section{Subjects}

Severe acute pancreatitis patients $(\mathrm{N}=77)$ hospitalized in the Wuxi Hospital of Integrated Traditional Chinese and Western Medicine in China during the period from March 2010 to August 2014 were enrolled in this study. They were randomly divided into a control group $(\mathrm{N}=36,21$ 
males, 15 females, aged 21-68 years), and an experimental group ( $\mathrm{N}=41,25$ males, 16 females, aged 22-65 years). Patients met the following inclusion criteria: 1) diagnosis of severe acute pancreatitis in accordance with the criteria of Combined Diagnosis and Treatment of Traditional Chinese Medicine and Western Medicine for Severe Acute Pancreatitis (Draft) (General Surgery Committee, Chinese Association of the Integration of Traditional and Western Medicine, 2007) with severe acute pancreatitis diagnostic criteria and persistent organ dysfunction ( $>48 \mathrm{~h}$, single organ or multi-organ) or local complications, such as necrosis, abscess, pseudocyst; 2) Acute Physiology and Chronic Health Evaluation II scoring system (APACHE II) score $\geq 8$ points; 3 ) willingness to receive combined treatment of traditional Chinese and Western medicine. The exclusion criteria were: 1) patients presented with trauma, pregnancy, hepatitis B surface antigen positive, severe cardiovascular disease, immunodeficiency, cancer, or end-stage liver or kidney diseases; 2) patients treated surgically, patients refusing or could not implement the therapeutic regimen. This study was conducted in accordance with the medical ethical standards approved by the Hospital Ethics Committee (Chinese Clinical Trial Registry, Registration No. ChiCTRTRC-09000610). All patients or their family members signed informed consents.

\section{Methods}

All subjects underwent the following treatments: blood samples were collected at 1, 3, 7, and 14 days after hospital admission to determine the blood levels of interleukin (IL)-6, IL-10, tumor necrosis factor (TNF)- $\alpha$, endotoxin levels, HLA-DR, Treg, Th1, and Th2 cells. Simultaneously, the urinary lactulose/mannitol ratio (LMR) was measured. Day 14 following hospital admission was considered the endpoint of the study, and the mortality was recorded in both groups.

\section{Therapeutic regimen}

All subjects received conventional therapy (enzymatic and acid inhibitory drugs, antiinflammatory drugs, and liquid management) and supportive treatment to support organ function in accordance with the previously published criteria (General Surgery Committee, Chinese Association of the Integration of Traditional and Western Medicine, 2007). In addition, the experimental group received modified Da Chengqi granules [11.2 g/bag (Jiangyin Tianjiang Pharmaceutical Co., Ltd. Wuxi, China); twice a day, two bags each time]. One bag of modified Da Chengqi granules was mixed with $200 \mathrm{~mL}$ boiled water and given orally or by gastric tube. An additional bag of modified Da Chengqi granules was mixed with $400 \mathrm{~mL}$ boiled water and given by enema.

\section{Determination of inflammatory cytokines, immunocytes, and endotoxin levels}

Venous blood $(5 \mathrm{~mL})$ was obtained from all patients with empty stomach on the morning of days $1,3,7$, and 14 following hospital admission, and placed separately into dry, heat-resistant test tubes. Within $2 \mathrm{~h}$ after serum isolation, samples used for the assay of inflammatory cytokines were stored at $-80^{\circ} \mathrm{C}$. Endotoxin levels were determined on the day of sample collection. Ethylenediaminetetraacetic acid (EDTA) was used to detect immunocytes on the day of sample collection. IL-6, IL-10, and TNF- $\alpha$ levels were measured using enzymelinked immunosorbent assay (ELISA) kit (eBioscience, San Diego, CA, USA). Endotoxin levels were determined using a chromogenic endpoint Tachypleus Amebocyte Lysate (TAL) kit (Shanghai Yihua Medical Technology Co., Ltd., Shanghai, China) and read using a 
SpectraMax 340 microplate reader (Molecular Devices Inc., Sunnyvale, CA, USA). HLA-DR (CD14+/HLA-DR+), Treg (CD4+/CD25+/Foxp3+), Th1 $(\mathrm{CD} 4+/$ IFN- $\gamma+)$ and Th2 (CD4+/ IL-4+) cells were detected by FACSCalibur flow cytometry (BD, Franklin Lake, NJ, USA), according to the manufacturer's instructions.

\section{Detection of urinary LMR}

All patients were administered $20 \mathrm{~mL}$ lactulose/mannitol orally, after urination, on the morning of days $1,3,7$, and 14 following hospital admission. Thereafter, 6-hour urine (3 $\mathrm{mL}$ ) was placed at $-80^{\circ} \mathrm{C}$ for future use. High-pressure liquid chromatography (Model 1100, Agilent, Palo Alto, CA, USA) was used to determine urinary LMR.

\section{Statistical methods}

Quantitative data are reported as means $\pm \mathrm{SD}$, and analyzed using the SPSS 16.0 software (SPSS Inc., Chicago, IL, USA). Intergroup comparisons at the same time point were performed using the unpaired $t$-test, whereas intragroup comparisons at different time points were performed using paired sample $t$-test. Qualitative data were compared using chi-square $\left(\chi^{2}\right)$ test. A value of $\mathrm{P}<0.05$ was considered statistically significant.

\section{RESULTS}

\section{Quantitative analysis and clinical prognosis of the subjects}

Of all the 77 early severe acute pancreatitis patients, 59 completed the 14-day therapy. Five patients quit automatically within the second week of the study because of their stable condition. Seven patients quit in the first 3 days due to a therapy change. Five deaths occurred on days $3-7,1$ on days $7-14$, and 3 on days $14-28$. The total mortality rate was $11.69 \%(9 / 77)$, which was significantly lower in the experimental group $[4.88 \%(2 / 41)]$ as compared with the control group $\left[19.44 \%(7 / 36) ; \chi^{2}=3.940, \mathrm{P}<0.05\right]$.

\section{Comparison of the baseline data between experimental and control groups}

We examined the 77 severe acute pancreatitis patients to establish baseline data. No significant differences regarding the age (47.68 \pm 13.46 vs $44.86 \pm 11.14$ years; $t=0.734$, $\mathrm{P}=0.467$ ) or gender (male/female: $25 / 16$ vs $21 / 15, \chi^{2}=0.056, \mathrm{P}=0.814$ ) were detected between experimental and control groups (all $\mathrm{P}>0.05$ ). Similarly, we did not find significant differences in immunological indices, urinary LMR, or endotoxin levels on day 1 between experimental and control groups (all $\mathrm{P}>0.05$; Table 1).

Table 1. Comparison of immunological indices, urinary LMR, and endotoxin levels between experimental and control groups at day 1.

\begin{tabular}{l|c|c|c|c|c|c|c|c|c}
\hline Groups & Cases & IL-6 $(\mathrm{pg} / \mathrm{mL})$ & $\mathrm{IL}-10(\mathrm{pg} / \mathrm{mL})$ & TNF- $\alpha(\mathrm{pg} / \mathrm{mL})$ & HLA-DR $(\%)$ & Treg $(\%)$ & TH1/TH2 $(\%)$ & L/M (\%) & ET (EU/mL) \\
\hline Control group & 36 & $39.21 \pm 6.68$ & $16.56 \pm 3.62$ & $75.18 \pm 12.06$ & $67.20 \pm 15.15$ & $8.56 \pm 0.89$ & $6.51 \pm 0.67$ & $0.54 \pm 0.17$ & $0.42 \pm 0.12$ \\
\hline Experimental group & 41 & $41.08 \pm 7.04$ & $17.66 \pm 3.37$ & $74.29 \pm 12.21$ & $66.54 \pm 14.91$ & $8.45 \pm 1.02$ & $6.44 \pm 0.76$ & $0.52 \pm 0.13$ & $0.43 \pm 0.14$ \\
\hline$t$ value & & 0.867 & 1.006 & 0.233 & 0.142 & 0.373 & 0.308 & 0.486 & 0.154 \\
\hline P value & & 0.391 & 0.320 & 0.817 & 0.888 & 0.711 & 0.760 & 0.630 & 0.879 \\
\hline
\end{tabular}




\section{Difference in inflammatory cytokines levels between experimental and control groups}

IL-6, IL-10, and TNF- $\alpha$ levels were significantly lower during the period between day 7 and day 14 than on day 1 in both the experimental and control groups (all $\mathrm{P}<0.01$ ). However, this difference was not visible prior to day 7 (all P > 0.05). Moreover, IL-6, IL-10, and TNF- $\alpha$ levels were significantly lower in the experimental group by day $14(\mathrm{P}<0.01$ or $\mathrm{P}<0.05$; Figure 1).
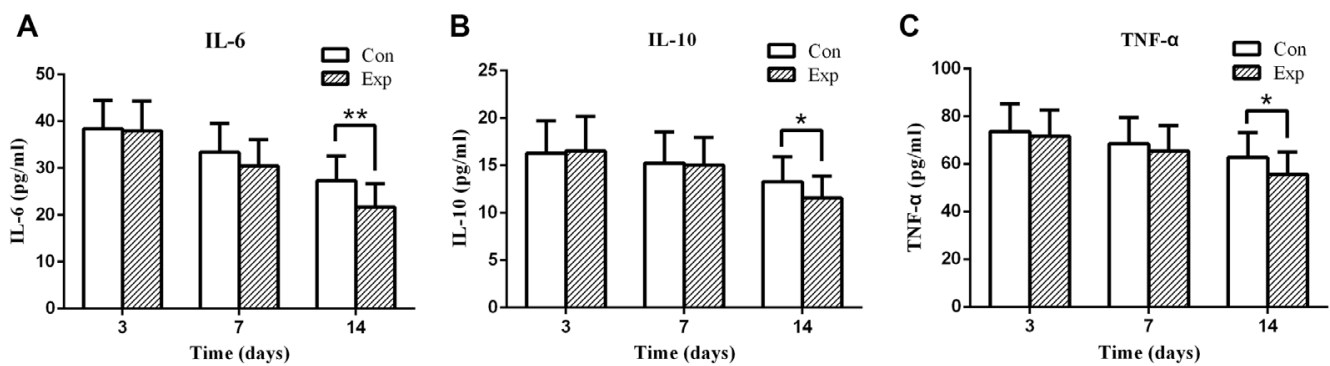

Figure 1. Comparison of IL-6, IL-10, and TNF- $\alpha$ levels in experimental and control groups with time of therapy. IL6, IL-10, and TNF- $\alpha$ levels were detected at 3, 7, and 14 days after hospital admission. A. IL-6. B. IL-10. C. TNF- $\alpha$. Data are given as the means $\pm \mathrm{SD}(\mathrm{N}=41$ patients). $* \mathrm{P}<0.05, * * \mathrm{P}<0.01$ compared with control group.

\section{Changes in HLA-DR-positive cells, Treg cells, and Th1/Th2 ratio in the experimental and control groups}

The percentage of HLA-DR-positive cells increased gradually in the experimental and control groups after 14 days of treatment (Figures 2 and 3) (all $\mathrm{P}<0.01$ ). No significant difference was observed by day 7 (all $\mathrm{P}>0.05$ ), however, the ratio of HLA-DR-positive cells was significantly higher in the experimental group relative to the control group on day $14(\mathrm{P}<$ 0.05). The percentage of Treg cells was found to be the same on days 3 and 7 in the experimental and control groups (all $\mathrm{P}<0.01)$, but decreased in the experimental group by day $14(\mathrm{P}<0.01)$. Although no significant difference in the percentage of Treg cells was detected between the two groups on day 7 (all $\mathrm{P}>0.05$ ), however, their percentage decreased in the experimental group on day $14(\mathrm{P}<0.01)$. Flow cytometry revealed that Th1/Th2 ratio decreased on days 3 and 7 in the experimental group (all $\mathrm{P}<0.01)$. However, it increased on day $14(\mathrm{P}<0.01$ or 0.05$)$.
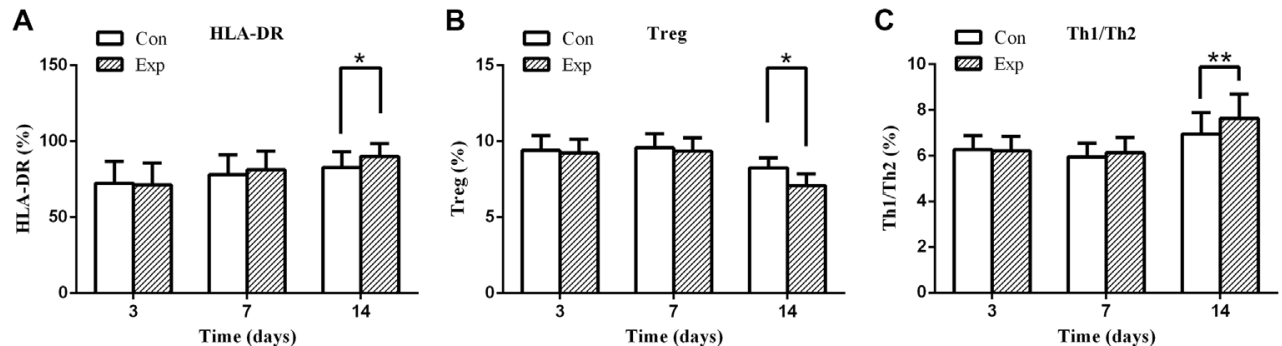

Figure 2. Comparison of HLA-DR, Treg, and Th1/Th2 ratio in the experimental and control groups with prolonged time. HLA-DR, Treg, and Th1/Th2 ratio were detected at 3, 7, and 14 days after hospital admission. A. HLA-DR. B. Treg. C. $\mathrm{Th} 1 / \mathrm{Th} 2$ ratio. Data are reported as means $\pm \mathrm{SD}\left(\mathrm{N}=41\right.$ patients). ${ }^{*} \mathrm{P}<0.05, * * \mathrm{P}<0.01$ compared with control group. 
Control group
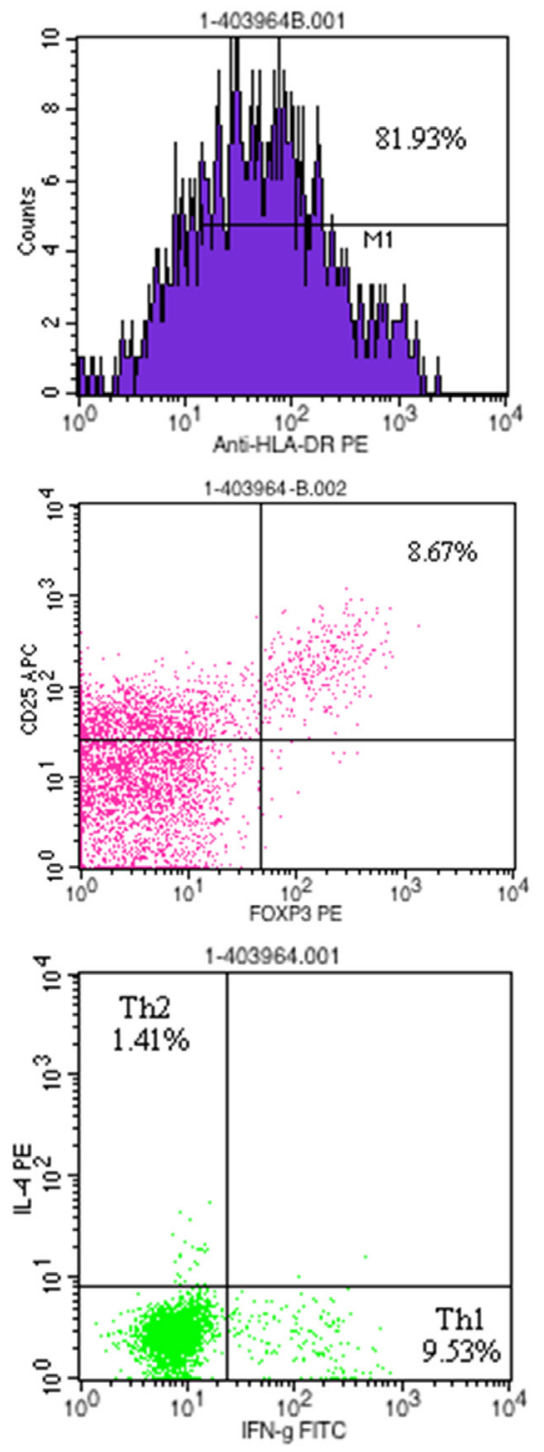

Experimental group
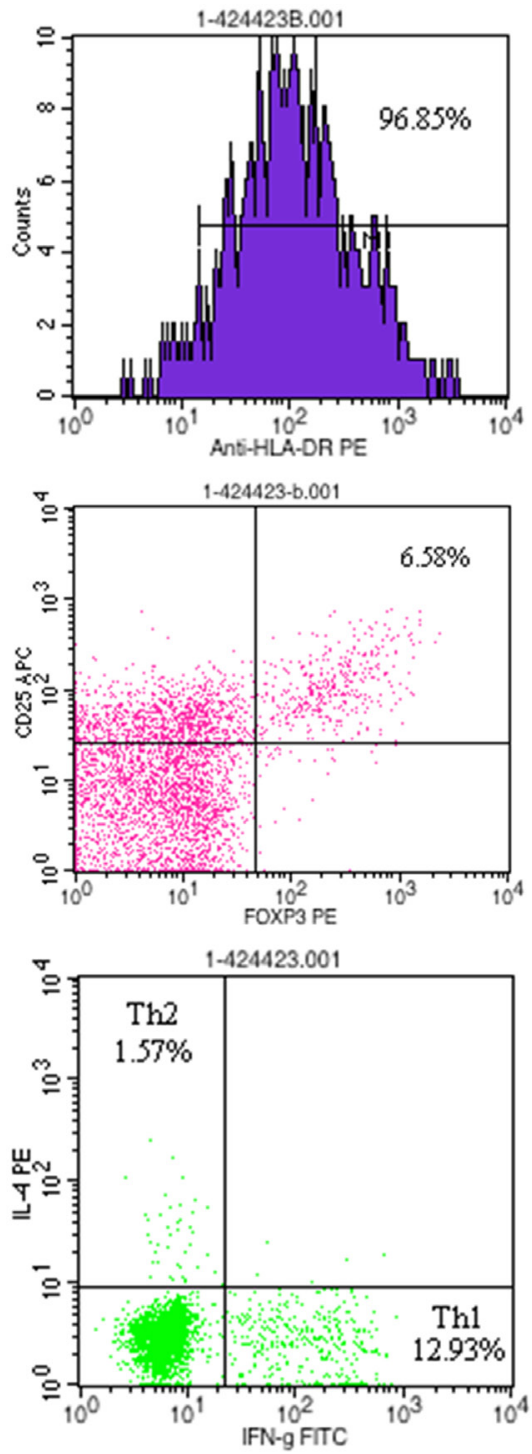

Figure 3. Flow cytometry of percent HLA-DR, percent Treg, and Th1/Th2 ratio with their average value at the experimental end point (day 14).

\section{Urinary LMR and endotoxin changes in experimental and control groups}

Urinary LMR and blood endotoxin levels were not significantly different between the experimental and control groups on days 7 and 14 (all $\mathrm{P}>0.05$ ). However, both were lower in the experimental group on day 14 (all $\mathrm{P}<0.01$; Figure 4). 

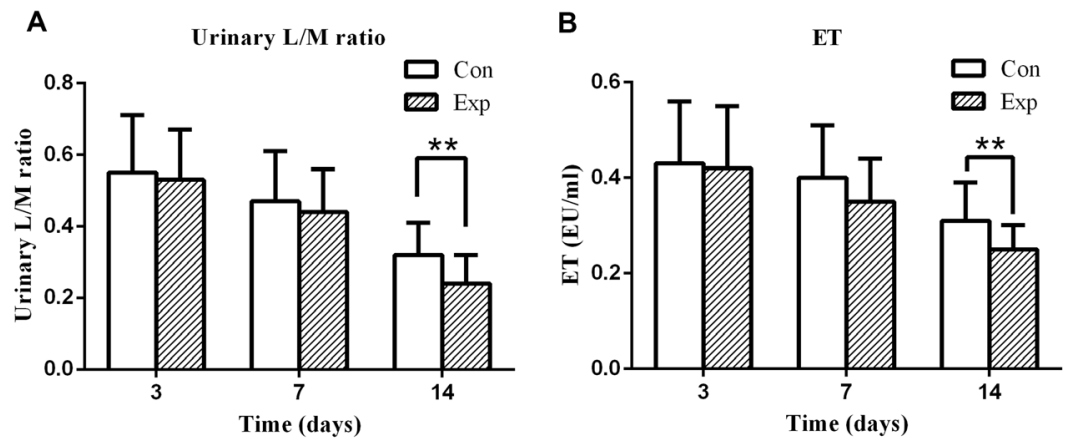

Figure 4. Comparison of the urinary LMR and blood endotoxin levels in experimental and control groups with prolonged treatment. Urinary L/M ratio, blood endotoxin levels (ET) were detected at 3, 7, and 14 days after hospital admission. A. Urinary L/M ratio. B. ET. Data are reported as means $\pm \mathrm{SD}(\mathrm{N}=41$ patients $) .{ }^{*} \mathrm{P}<0.01$ compared with control group.

\section{Adverse reactions}

In the experimental group, two patients experienced abdominal distension, and one patient presented with mild diarrhea, which decreased after expectant treatment. There were no medication-related adverse reactions in the control group.

\section{DISCUSSION}

Cell-mediated immunity is mainly mediated by the direct killing effect of activated-T lymphocytes and the synergistic killing effect of the cytokines released by these cells. In an immune response against an infection, cell- mediated immunity is not only the main defense mechanism against pathogens but also an important factor leading to immuneopathological injury. The T-helper (Th) cells, mainly Th1 and Th2 play a role in immunoregulation (Gong et al., 2014). The ratio of Th1/Th2 cells seems to be stable. These cells secrete many immune mediators including IL-6, IL-10 and play an important role in specific and non-specific immunity (Gong et al., 2012, 2013). The main functional role of regulatory $\mathrm{T}$ (Treg) cells involves secretion of anti-inflammatory mediators such as IL-10 and TGF- $\beta$, thus they regulate autoimmune responses. HLA-DR, a MHC class II molecule, is a protein that must be expressed by antigen-presenting cells in order to present antigens to $\mathrm{T}$ cells. The expression level of HLA-DR is a sensitive index that reflects the immune function in patients with infectious diseases. Patients with low level of HLA-DR ${ }^{+}$cells suffer from decreased immune function or even "immune paralysis" state, and consequently, the risk of infection is increased. IL-6, IL10 , and TNF- $\alpha$ are the most important mediators of immune responses in vivo.

Severe acute pancreatitis patients experience apparent immunological imbalance and intestinal mucosal dysfunction. This may be due to peripancreatic fluid collections, elevated plasma lipid levels, alcohol consumption, trauma, autoimmunity triggered by pancreatic antigens, or inflammatory reaction mediated by inflammatory cytokines, both of which may trigger a "waterfall-like" cascade reaction of inflammatory mediators (Bansal et al., 2011; Susantitaphong et al., 2013). This clinically manifests as a strong non-infectious systemic inflammatory reaction, resulting in a severe autoimmune attack against multiple organs (Bansal 
et al., 2011; Susantitaphong et al., 2013). As the patient's condition worsens, peripheral blood $\mathrm{T}$ and B lymphocytes and HLA-DR+ cells decrease, Treg cells increase, and Th1/Th2 cell ratio decreases, resulting in immune suppression and severe complications (Pietruczuk et al., 2006; Malleo et al., 2007; Dabrowski et al., 2008). During this time, they experience severe stress, intestinal paralysis, defective pancreatic secretory function, impaired immune defenses, intestinal mucosal lesions, and translocation of bacteria and endotoxins to the bloodstream leading to endotoxemia and systemic inflammatory reaction (Al-Bahrani et al., 2010; Sharma et al., 2011). Therefore, it is important to restore the immune balance during treatment of severe acute pancreatitis.

The present study used a combination treatment of traditional Chinese and Western medicine. In addition, to complement treatment, patients received enzymatic and acid suppressive drugs, anti-inflammatory medications, liquid management, and organ support, and were administered modified Da Chengqi granules. These granules are composed of snakegourd fruit, immature orange fruit, rhubarb, pinellia tuber, Magnoliaeofficinalis, and goldthread. These granules regulate the functions of lung, stomach, and large intestine. Snakegourd fruit moistens the lungs and intestine, eliminates sputum, and treats stasis. Snakegourd root, a main component of snakegourd fruit, has immunoregulatory function that helps resist hypoxia, activates immunocytes, and increases the ability to defend against infections (Zhang et al., 2009; Xu et al., 2010; Yan et al., 2012). Immature orange fruit relieves constipation, displays anti-inflammatory and antioxidant effects (Menichini et al., 2011; Kang et al., 2013; Yu et al., 2013), promotes gut hormone secretion, and improves gastrointestinal motility (Wu et al., 2011; Lyu and Lee, 2013). Rhubarb and goldthread clear away heat-evil and expel superficial evils, and thus have a positive therapeutic impact on pancreatitis (Wu et al., 2000; Wang et al., 2007; Li et al., 2009). Moreover, they decrease IL-6 and TNF- $\alpha$ levels and reduce the pathological lesions in acute pancreatitis patients (Zhang et al., 2005). Goldthread relieves endotoxin-induced fever, reduces IL-6, TNF- $\alpha$, and interferon- $\beta$ levels (Lu et al., 2007; Kim et al., 2010; Zhang et al., 2011; Choi et al., 2013). Pinellia tuber and Magnoliaeofficinalis have anxiolytic and antiemetic effects. Furthermore, they help remove qi stagnation, regulate gastrointestinal function and cellular immunity (Lee and Cho, 2009; Park et al., 2010; Weng et al., 2012), protect gastric mucosa (Wu et al., 2010), and reduce the expression of inflammatory mediators. Taken together, the theory of "the lung and the large intestine being interiorexteriorly related" appears to work. The lung and intestine are treated in the same manner, which involves regulation of immunity, improvement of lung oxygenation, maintenance of the intestinal mucosa, and thus, promoting the recovery.

In this study, TNF- $\alpha$, IL-10, and IL-6 levels decreased in patients in the experimental group. TNF- $\alpha$ and IL- 6 are proinflammatory cytokines while IL-10 is an anti-inflammatory cytokine. Thus, humoral immunity gradually returned to normal. TNF- $\alpha$, IL-10, and IL-6 levels were significantly lower in the experimental group than those in the control group by day 14 (all $\mathrm{P}<0.05$ ), indicating that modified $D a$ Chengqi granules gradually improved patient humoral immunity. During treatment, the HLA-DR ${ }^{+}$cells percentage gradually increased in both groups, with Treg cells increasing over the first 7 days, but decreasing again by day 14 . Th1/Th2 ratio decreased steadily over the first 7 days, but increased again by day 14. HLA$\mathrm{DR}^{+}$cells serve as antigen-presenting cells. Treg cells inhibit cellular immunity, and regulate Th1/Th2 ratio, which suggests that antigen presentation was suppressed in the first 7 days, and relieved by day 14 . These changes were more significant in the experimental group than in the control group (all $\mathrm{P}<0.05$ ), suggesting that modified $D a$ Chengqi granules improve cellular 
immunity in severe acute pancreatitis patients. Urinary LMR and blood endotoxin levels were gradually reduced during treatment (all $\mathrm{P}<0.01$ ), indicating that intestinal mucosal injury gradually improved with the granules administration. Due to modified Da Chengqi granulesmediated improved immunologic function, death rate in the experimental group was lower than that in the control group during the first 28 days. Previous studies confirmed that rhubarb noticeably reduces serum amylase, cytokines, and endotoxin levels in rat models of severe acute pancreatitis, so it inhibits excessive inflammatory reaction, regulates immunity, and limits the damage to the pancreatic gland and intestinal barrier (Wang et al., 2008, 2010). This is consistent with our findings.

In summary, combined treatment of traditional Chinese and Western medicine improves humoral immunity and cellular immunity, contributes to the recovery of intestinal mucosal function, and decreases the death rate in severe acute pancreatitis patients.

\section{Conflicts of interest}

The authors declared no conflict of interest.

\section{ACKNOWLEDGMENTS}

Research supported by the National Key Basic Research Development Project (“973” Project) in China (\#2009CB522703).

\section{REFERENCES}

Al-Bahrani AZ, Darwish A, Hamza N, Benson J, et al. (2010). Gut barrier dysfunction in critically ill surgical patients with abdominal compartment syndrome. Pancreas 39: 1064-1069. http://dx.doi.org/10.1097/MPA.0b013e3181da8d51

Bansal D, Bhalla A, Bhasin DK, Pandhi P, et al. (2011). Safety and efficacy of vitamin-based antioxidant therapy in patients with severe acute pancreatitis: a randomized controlled trial. Saudi J. Gastroenterol. 17: 174-179. http:// dx.doi.org/10.4103/1319-3767.80379

Barie PS, Hydo LJ, Pieracci FM, Shou J, et al. (2009). Multiple organ dysfunction syndrome in critical surgical illness. Surg. Infect. 10: 369-377. http://dx.doi.org/10.1089/sur.2009.9935

Choi YY, Kim MH, Cho IH, Kim JH, et al. (2013). Inhibitory effect of Coptis chinensis on inflammation in LPS-induced endotoxemia. J. Ethnopharmacol. 149: 506-512. http://dx.doi.org/10.1016/j.jep.2013.07.008

Dabrowski A, Osada J, Dabrowska MI and Wereszczynska-Siemiatkowska U (2008). Monocyte subsets and natural killer cells in acute pancreatitis. Pancreatology 8: 126-134. http://dx.doi.org/10.1159/000123605

Dambrauskas Z, Giese N, Gulbinas A, Giese T, et al. (2010). Different profiles of cytokine expression during mild and severe acute pancreatitis. World J. Gastroenterol. 16: 1845-1853. http://dx.doi.org/10.3748/wjg.v16.i15.1845

Fang SD, Chen RT and Wei BY (2007). An overview on modernization of the traditional Chinese medicine. ZhongCaoyao 5: 641-646.

General Surgery Committee, Chinese Association of the Integration of Traditional and Western Medicine (2007). Practical guidelines for management of severe acute pancreatitis with integrated Chinese traditional and Western medicine (Draft). Zhongguo Wei Zhong Bing Ji Jiu Yi Xue 19: 448-451.

Gong F, Song S, Lv G, Pan Y, et al. (2012). Human leukocyte antigen E in HCMV infection: friend or foe? Acta Biochim. Biophys. Sin. 44: 551-554. http://dx.doi.org/10.1093/abbs/gms032

Gong F, Ding L, Jiang D, Zhang C, et al. (2013). Association of human leukocyte antigen E polymorphism with human cytomegalovirus reactivation in Chinese burn patients. Acta Biochim. Biophys. Sin. 45: 982-984. http://dx.doi. org/10.1093/abbs/gmt103

Gong F, Su Q, Jiang D, Chen J, et al. (2014). High frequency of circulating follicular helper T cells in patients with bronchial asthma. Clin. Lab. 60: 963-968.

Kang GJ, Han SC, Ock JW, Kang HK, et al. (2013). Anti-Inflammatory Effect of Quercetagetin, an Active Component 
of Immature Citrus unshiu, in HaCaT Human Keratinocytes. Biomol. Ther. (Seoul) 21: 138-145. http://dx.doi. org/10.4062/biomolther.2013.001

Kim JM, Jung HA, Choi JS, Min BS, et al. (2010). Comparative analysis of the anti-inflammatory activity of Huanglian extracts in lipopolysaccharide-stimulated RAW264.7 murine macrophage-like cells using oligonucleotide microarrays. Arch. Pharm. Res. 33: 1149-1157. http://dx.doi.org/10.1007/s12272-010-0803-3

Kylänpää ML, Repo H and Puolakkainen PA (2010). Inflammation and immunosuppression in severe acute pancreatitis. World J. Gastroenterol. 16: 2867-2872. http://dx.doi.org/10.3748/wjg.v16.i23.2867

Lee SY and Cho JY (2009). Inhibitory effects of honokiol on LPS and PMA-induced cellular responses of macrophages and monocytes. BMB Rep. 42: 574-579. http://dx.doi.org/10.5483/BMBRep.2009.42.9.574

Li ZF, Xia XM, Huang C, Zhang S, et al. (2009). Emodin and baicalein inhibit pancreatic stromal derived factor-1 expression in rats with acute pancreatitis. HBPD INT 8: 201-208.

Lu JS, Liu YQ, Li M, Li BS, et al. (2007). Protective effects and its mechanisms of total alkaloids from rhizome Coptischinensis on Helicobacter pylori LPS induced gastric lesion in rats. ZhongguoZhong Yao ZaZhi. 13: 1333-1336.

Lyu JH and Lee HT (2013). Effects of dried Citrus unshiu peels on gastrointestinal motility in rodents. Arch. Pharm. Res. 36: 641-648. http://dx.doi.org/10.1007/s12272-013-0080-z

Malleo G, Mazzon E, Siriwardena AK and Cuzzocrea S (2007). Role of tumor necrosis factor-alpha in acute pancreatitis: from biological basis to clinical evidence. Shock 28: 130-140. http://dx.doi.org/10.1097/shk.0b013e3180487ba1

Menichini F, Loizzo MR, Bonesi M, Conforti F, et al. (2011). Phytochemical profile, antioxidant, anti-inflammatory and hypoglycemic potential of hydroalcoholic extracts from Citrus medica L. cv Diamante flowers, leaves and fruits at two maturity stages. Food Chem. Toxicol. 49: 1549-1555. http://dx.doi.org/10.1016/j.fct.2011.03.048

Park JW, Ryu B, Yeo I, Jerng UM, et al. (2010). Banha-sasim-tang as an herbal formula for the treatment of functional dyspepsia: a randomized, double-blind, placebo-controlled, two-center trial. Trials 11: 83. http://dx.doi. org/10.1186/1745-6215-11-83

Pietruczuk M, Dabrowska MI, Wereszczynska-Siemiatkowska U and Dabrowski A (2006). Alteration of peripheral blood lymphocyte subsets in acute pancreatitis. World J. Gastroenterol. 12: 5344-5351. http://dx.doi.org/10.3748/wjg.v12. i33.5344

Sharma B, Srivastava S, Singh N, Sachdev V, et al. (2011). Role of probiotics on gut permeability and endotoxemia in patients with acute pancreatitis: a double-blind randomized controlled trial. J. Clin. Gastroenterol. 45: 442-448. http://dx.doi.org/10.1097/MCG.0b013e318201f9e2

Shen Y, Cui N, Miao B and Zhao E (2011). Immune dysregulation in patients with severe acute pancreatitis. Inflammation 34: 36-42. http://dx.doi.org/10.1007/s10753-010-9205-4

Susantitaphong P, Perianayagam MC, Tighiouart H, Liangos O, et al. (2013). Tumor necrosis factor alpha promoter polymorphism and severity of acute kidney injury. Nephron Clin. Pract. 123: 67-73. http://dx.doi. org $/ 10.1159 / 000351684$

Vlada AC, Schmit B, Perry A, Trevino JG, et al. (2013). Failure to follow evidence-based best practice guidelines in the treatment of severe acute pancreatitis. HPB 15: 822-827. http://dx.doi.org/10.1111/hpb.12140

Wang CH, Gao ZQ, Ye B, Cai JT, et al. (2007). Effect of emodin on pancreatic fibrosis in rats. World J. Gastroenterol. 13: 378-382. http://dx.doi.org/10.3748/wjg.v13.i3.378

Wang G, Sun B, Gao Y, Meng QH, et al. (2008). An experimental study of emodin assisted early enteral nutrition for the treatment of severe acute pancreatitis. Hepatogastroenterology 55: 33-40.

Wang G, Sun B, Zhu H, Gao Y, et al. (2010). Protective effects of emodin combined with danshensu on experimental severe acute pancreatitis. Inflamm. Res. 59: 479-488. http://dx.doi.org/10.1007/s00011-009-0152-1

Weng TI, Wu HY, Chen BL and Liu SH (2012). Honokiol attenuates the severity of acute pancreatitis and associated lung injury via acceleration of acinar cell apoptosis. Shock 37: 478-484. http://dx.doi.org/10.1097/SHK.0b013e31824653be

Wu H, Jing Z, Tang X, Wang X, et al. (2011). To compare the efficacy of two kinds of Zhizhu pills in the treatment of functional dyspepsia of spleen-deficiency and qi-stagnation syndrome: a randomized group sequential comparative trial. BMC Gastroenterol. 11: 81. http://dx.doi.org/10.1186/1471-230X-11-81

Wu JX, Xu JY and Yuan YZ (2000). Effect of emodin and sandostatin on metabolism of eicosanoids in acute necrotizing pancreatitis. World J. Gastroenterol. 6: 293-294.

Wu ZJ (2004). Anti-infective Treatment with Chinese Traditional Medicine. Chin. ZhonghuaYiyuanGanranxueZazhi. 11: 1296-1297.

Wu ZX, He LG, Tan DQ and Zhou L (2010). Effects of BanxiaXiexin decoction and its divisions on serum IFN- $\gamma$ in mice infected Hp. Hunan ZhongyiyaoDaxueXuebao. 005: 23-25.

Xu S, Zhao G, Tu J, Gu S, et al. (2010). Immunocompetence effects of polysaccharide of snakegourd root on human peripheral blood mononuclear cells in vitro. Zhongguo Zhong Yao Za Zhi 35: 745-749. 
Yan R, Zhong W, Zhu Y and Zhang X (2012). Trichosanthin-stimulated dendritic cells induce a type 2 helper T lymphocyte response through the OX40 ligand. J. Investig. Allergol. Clin. Immunol. 22: 491-500.

Yu MW, Lou SN, Chiu EM and Ho CT (2013). Antioxidant activity and effective compounds of immature calamondin peel. Food Chem. 136: 1130-1135. http://dx.doi.org/10.1016/j.foodchem.2012.09.088

Zhang Q, Piao XL, Piao XS, Lu T, et al. (2011). Preventive effect of Coptis chinensis and berberine on intestinal injury in rats challenged with lipopolysaccharides. Food Chem. Toxicol. 49: 61-69. http://dx.doi.org/10.1016/j.fct.2010.09.032

Zhang XP, Li ZF, Liu XG, Wu YT, et al. (2005). Effects of emodin and baicalein on rats with severe acute pancreatitis. World J. Gastroenterol. 11: 2095-2100. http://dx.doi.org/10.3748/wjg.v11.i14.2095

Zhang XX, Wang YP, Wang YF and Chen SB (2009). Effect of Pericarpium trichosanthis on Immunological Function in Immunosuppressive Mice Induced by Cyclophosphamide. Zhongguo Yaofang. 9: 648-650. 